\title{
Characterizing the risk of respiratory syncytial virus in infants with older siblings: a population-based birth cohort study
}

\author{
P. JACOBY ${ }^{1 *}$, K. GLASS ${ }^{2}$ AND H. C. MOORE ${ }^{1}$ \\ ${ }^{1}$ Wesfarmers Centre of Vaccines and Infectious Diseases, Telethon Kids Institute, The University of Western \\ Australia, Perth, Australia \\ ${ }^{2}$ National Centre for Epidemiology and Population Health, The Australian National University, Canberra, \\ Australia
}

Received 30 May 2016; Final revision 23 August 2016; Accepted 9 October 2016; first published online 8 November 2016

\section{SUMMARY}

From a population-based birth cohort of 245249 children born in Western Australia during 1996-2005, we used linkage of laboratory and birth record datasets to obtain data including all respiratory syncytial virus (RSV) detections during infancy from a subcohort of 87981 singleton children born in the Perth metropolitan area from 2000 to 2004. Using log binomial regression, we found that the risk of infant RSV detection increases with the number of older siblings, with those having $\geqslant 3$ older siblings experiencing almost three times the risk (relative risk $2 \cdot 83,95 \%$ confidence interval 2.46-3.26) of firstborn children. We estimate that $45 \%$ of the RSV detections in our subcohort were attributable to infection from an older sibling. The sibling effect was significantly higher for those infants who were younger during the season of peak risk (winter) than those who were older. Although older siblings were present in our cohort, they had very few RSV detections which could be temporally linked to an infant's infection. We conclude that RSV infection in older children leads to less severe symptoms but is nevertheless an important source of infant infection. Our results lend support to a vaccination strategy which includes family members in order to provide maximum protection for newborn babies.

Key words: Epidemiology, household, respiratory syncytial virus, vaccine policy development.

\section{INTRODUCTION}

Respiratory syncytial virus (RSV) is a major cause of acute lower respiratory infection (ALRI) in young children with a global estimate of over 3 million hospitalizations and $66000-199000$ deaths annually in children aged $<5$ years attributable to the virus [1]. Bronchiolitis is the most common ALRI in children aged $<1$ year, with a recent Italian cohort study estimating a hospital admission rate of 50.4/1000 infants

\footnotetext{
* Author for correspondence: A/Professor P. Jacoby, Telethon Kids Institute, PO Box 855, West Perth, WA 6872, Australia. (Email: Peter.Jacoby@telethonkids.org.au)
}

[2]. Bronchiolitis is caused by RSV in a reported $75 \%$ of cases [3].

RSV infection in temperate regions typically displays a seasonal pattern with a distinct peak in numbers during winter $[4,5]$. Although RSV infection occurs in all age groups [6,7], the risk of severe RSV-related respiratory disease after infection is highest in children, in particular young infants $[8,9]$. As yet there is no licensed vaccine targeting RSV but considerable progress is being made with a phase 3 clinical trial launched in 2015 of a RSV F nanoparticle vaccine in pregnant women [10].

Preterm birth has been identified as a major factor increasing the risk of RSV infection in infants [2, 11, 12]. 
However, the majority of children hospitalized with RSV infection are previously healthy term infants $[8$, 13]. A literature review of case-control and cohort studies investigating the role of non-medical risk factors for severe RSV-related illness [14] concluded that male sex, young age, birth during the first half of the RSV season, crowding, presence of siblings and daycare attendance were all significant risk factors for the development of RSV-related ALRI.

A number of household studies have reported varying estimates of the source of RSV infection. A cohort study in rural Kenya [15] estimated that $54 \%$ of infant RSV infections were acquired from within the household with older children being responsible for $73 \%$ of the household-acquired infections. A recent Finnish study [16] recruited family members of children hospitalized with RSV infection and estimated that $58 \%$ of the infections were acquired from household members with $50 \%$ of these acquired from siblings, $82 \%$ of the RSV-positive family members displayed some respiratory symptoms. In a similar British study [17] only $38 \%$ of infants were confirmed as the secondary case in the household with $57 \%$ of these infections originating from siblings, $50 \%$ of the RSV-positive family members reported no symptoms.

A recent population-based birth cohort study [18] investigated risk factors for acute RSV-related bronchiolitis admissions but this did not consider the influence of siblings. Our study is the first to investigate the effect of older siblings on risk of infant acute RSV-related illness in the setting of a population birth cohort using laboratory-confirmed identification of RSV. In addition to estimating the proportion of RSV infection attributable to older siblings, our data enable us to identify older siblings in the cohort and to ascertain any RSV detections from within the household which are likely to be associated with the infant's illness.

\section{METHODS}

\section{Data sources}

We had access to extensive population-based linked data including statewide routinely collected laboratory data through the Western Australian Data Linkage System (WADLS). Demographic, perinatal, clinical and laboratory data collected from administrative datasets were linked following a best practice protocol in order to maintain individuals' privacy [19].
Western Australia covers $\sim 2.5$ million $\mathrm{km}^{2}$ and has a population of 2.2 million. About three-quarters of Western Australia's population reside in the Perth metropolitan area in the south-west of the state which has a temperate climate. There is one dedicated tertiary-level paediatric teaching hospital in Western Australia, Princess Margaret Hospital for Children $(\mathrm{PMH})$ which is located in Perth. At PMH, it is standard practice to collect nasopharyngeal aspirates for respiratory virus detection on all children admitted to hospital with ALRI [20]. A recommendation for respiratory pathogen testing is in place at other smaller metropolitan and non-metropolitan hospitals across Western Australia.

Previously we extracted data from WADLS on 245 249 singleton live births in Western Australia between 1996 and 2005 from the Midwives' Notification System, Birth and Death Register and the Hospital Morbidity Database System. In brief, our linked dataset contains information on birth and pregnancy details, demographics and hospitalization episodes for ALRI between 1996 and 2005 [21]. Aboriginal children, who account for $7 \cdot 1 \%$ of the birth cohort, are identified as such if their mother is identified as Aboriginal or Torres Strait Islander on the Midwives' Notification System database or the child is identified as Aboriginal or Torres Strait Islander on their hospitalization, birth or death records.

We also extracted data from the PathWest Laboratory Database concerning routine detections of respiratory viruses and bacteria. PathWest Laboratory Medicine Western Australia (PathWest) is the government-funded public laboratory service, consists of all public pathology laboratories in Western Australia and carries out a full range of diagnostic testing for infectious diseases. Details of the component datasets within the PathWest Laboratory Database are given elsewhere [22]. Laboratory data were available from 2000 to 2005 for all children in the birth cohort for ages $0-9$ years. Respiratory samples received at PathWest for viral testing are routinely investigated for RSV, influenza viruses A and B, adenoviruses and parainfluenza virus types $1-3$. RSV was detected by either immunofluorescent antigen detection, polymerase chain reaction and/or viral culture. Testing methods for RSV did not change between 2000 and 2005. The use of these population-based linked data was approved by the Western Australian Department of Health Human Research Ethics Committee and the Western Australian Aboriginal Health Ethics Committee. 


\section{Statistical methods}

Within the larger cohort, we created a subcohort of singleton children born between 2000 and 2004 to conduct the older sibling analysis. We conducted logbinomial regression to estimate the relative risks with $95 \%$ confidence intervals for RSV identification in infants with older siblings compared to firstborn children. Birth order was a categorical predictor coded as $1,2,3$ and $\geqslant 4$. The regression model was adjusted for the following potential confounders: gender, preterm birth ( $<37$ weeks), birthweight, Aboriginal status, maternal age (five groups: <20, 20-24, 25-29, 30-34, $>35$ years) and maternal smoking during pregnancy. We used the adjusted relative risks to estimate the excess number of RSV cases attributable to older siblings using Kleinbaum's formula (see [23]). In order to assess whether any sibling effect varied with infant's age during the winter season, we subsequently included terms for birth season and its interaction with birth order in our regression model. Birth season was defined as spring (births in SeptemberNovember), summer (December-February), autumn (March-May) and winter (June-August) in keeping with Australia's climate. Adjusted probabilities of RSV detection for each combination of birth order and birth season were calculated. Analyses were conducted using SPSS v. 22 (IBM Corp., USA).

\section{RESULTS}

There were 4920 RSV identifications during 20002005 from 4557 individual children in the birth cohort. Of these identifications, $3349(68 \cdot 1 \%)$ were from children aged $<1$ year, $892(18 \cdot 1 \%)$ from children $1-<2$ years, $609(12 \cdot 4 \%)$ from children $2-<5$ years and 70 $(1 \cdot 4 \%)$ from children aged $\geqslant 5$ years. Identifications were predominately in the winter months with 3658 (74.4\%) occurring between 1 June and 31 August.

There were 87981 singleton children born during 2000-2004 in the Perth metropolitan area who survived to their first birthday and for whom we had complete data on the potential confounding variables listed above and all RSV identifications up to their first birthday. These children formed our subcohort for the older sibling analysis. One or more RSV identifications were experienced by $1977(2 \cdot 2 \%)$ children in the subcohort during their first year of life.

Crude incidence of RSV before 1 year of age according to the number of older siblings and other characteristics is summarized in Table 1 . Incidence rates increased from $1 \cdot 4 \%$ for firstborns to $3 \cdot 4 \%$ for children with $\geqslant 3$ older siblings.

Adjusted and unadjusted older sibling relative risks for RSV identification are shown in Table 2. The adjusted relative risks increase from 1.79 [95\% confidence interval (CI) 1.57-2.04] for those with one older sibling compared to firstborns, to 2.83 (95\% CI 2.46-3.26) for those with $\geqslant 3$ older siblings compared to firstborns. Application of Kleinbaum's formula gives an older sibling attributable fraction of 0.448 corresponding to an estimated 886/1977 RSV identifications being attributable to infection by an older sibling. However, there were only 29 instances where we were able to match an infant in the subcohort with an older sibling in the original birth cohort for a RSV identification in the same season using the unique identifier for each mother.

There was a significant $(P=0.02)$ birth order $\times$ birth season interaction effect when order was coded as first or subsequent birth. Figure 1 shows how the model-fitted probability of an RSV detection for each value of birth order varies by birth season. The older sibling effect is substantially more pronounced for those born during autumn (and therefore on average 3 months old at the time of peak winter risk) than it is for those born during spring who are on average 9 months old at the time of the winter peak. In firstborn children there is only a small variation in the likelihood of RSV detection rates according to the time of the year that the child is born.

\section{DISCUSSION}

We have shown, using total population data, a statistically significant increase in the risk of RSV detection for infants with older siblings compared to firstborns. This effect increases with the number of siblings and we estimate that the risk of RSV identification is increased almost threefold if the infant has $\geqslant 3$ older siblings. These results confirm the findings of previous household studies which highlighted the importance of family members in the transmission of RSV to infants.

The effect of siblings on the risk of RSV detection was significantly greater for children born shortly before the winter season who are the youngest at-risk group in our cohort. Interpretation of results for winter births is problematic as these births may have occurred before or after the peak RSV risk period. We also noted (Fig. 1) that for infants with no older siblings the rate of RSV detection does not vary 
Table 1. Characteristics of study children and identification of respiratory syncytial virus $(R S V)$ in first year of life

\begin{tabular}{|c|c|c|}
\hline Characteristic & $N$ & RSV identified (\%) \\
\hline \multicolumn{3}{|c|}{ No. of older siblings } \\
\hline 0 & 26730 & $367(1 \cdot 4)$ \\
\hline 1 & 27598 & $592(2 \cdot 1)$ \\
\hline 2 & 16466 & $438(2 \cdot 7)$ \\
\hline$\geqslant 3$ & 17187 & $580(3 \cdot 4)$ \\
\hline \multicolumn{3}{|l|}{ Gender } \\
\hline Male & 44993 & $1152(2 \cdot 6)$ \\
\hline Female & 42988 & $825(1 \cdot 9)$ \\
\hline \multicolumn{3}{|c|}{ Preterm (<37 weeks) } \\
\hline Yes & 5738 & $286(5 \cdot 0)$ \\
\hline No & 82243 & $1691(2 \cdot 1)$ \\
\hline \multicolumn{3}{|c|}{ Gestational smoking } \\
\hline Yes & 15138 & $523(3 \cdot 5)$ \\
\hline No & 72843 & $1454(2 \cdot 0)$ \\
\hline \multicolumn{3}{|c|}{ Aboriginal status } \\
\hline Yes & 3046 & $177(5 \cdot 8)$ \\
\hline No & 84935 & $1800(2 \cdot 1)$ \\
\hline \multicolumn{3}{|l|}{ Birth season } \\
\hline Spring & 22241 & $326(1 \cdot 5)$ \\
\hline Summer & 21641 & $448(2 \cdot 1)$ \\
\hline Autumn & 22441 & $697(3 \cdot 1)$ \\
\hline Winter & 21658 & $506(2 \cdot 3)$ \\
\hline \multicolumn{3}{|c|}{ Maternal age, years } \\
\hline$<20$ & 4185 & $158(3 \cdot 8)$ \\
\hline $20-24$ & 13228 & $394(3 \cdot 0)$ \\
\hline $25-29$ & 24819 & $560(2 \cdot 3)$ \\
\hline $30-34$ & 29292 & $535(1 \cdot 8)$ \\
\hline$\geqslant 35$ & 16457 & $330(2 \cdot 0)$ \\
\hline Total & 87981 & $1977(2 \cdot 2)$ \\
\hline
\end{tabular}

greatly with birth season indicating that, for this group, the risk of infection is broadly constant during the first year of life. The incidence of severe RSV infection is known to be highest in very young $(<3$ months) infants $[8,13]$ but our results suggest that this may only be the case for those with older siblings in the household.

We estimated that $45 \%$ of RSV infections in the infants in our subcohort were attributable to older siblings. All older siblings aged $<4$ years would be included in our original birth cohort and, if they were to experience an RSV infection leading to hospitalization for ALRI, we would expect an identification record in our data. However, we could find very few instances of a RSV detection in an older sibling which could be temporally linked to the infant's infection. We have recently conducted a validation of the laboratory data for respiratory infections obtained through data linkage against laboratory requests in
Table 2. Older siblings' relative risks for RSV detection in children aged $<1$ year

\begin{tabular}{lll}
\hline \hline $\begin{array}{l}\text { No. of older } \\
\text { siblings }\end{array}$ & $\begin{array}{l}\text { RR }(95 \% \mathrm{CI}) \\
\text { unadjusted }\end{array}$ & $\begin{array}{l}\text { RR }(95 \% \mathrm{CI}) \\
\text { adjusted* }\end{array}$ \\
\hline 0 & Ref. & Ref. \\
1 & $1 \cdot 56(1 \cdot 37-1 \cdot 78)$ & $1 \cdot 79(1 \cdot 57-2 \cdot 04)$ \\
2 & $1 \cdot 94(1 \cdot 69-2 \cdot 22)$ & $2 \cdot 31(2 \cdot 01-2 \cdot 66)$ \\
$\geqslant 3$ & $2 \cdot 46(2 \cdot 16-2 \cdot 80)$ & $2 \cdot 83(2 \cdot 46-3 \cdot 26)$ \\
\hline \hline
\end{tabular}

RSV, Respiratory syncytial virus; RR, relative risk; CI, confidence interval.

* Adjusted for gender, Aboriginal status, preterm birth $(<37$ weeks), birthweight, maternal age $(<20,20-24,25-29,30$ $34, \geqslant 35)$ and gestational smoking

hospital medical charts and concluded that our data extraction for linkage is likely to have missed some routine laboratory records for respiratory pathogens [24]. These missing records could partially explain why we were able to identify few instances of a RSV detection in an older sibling. Another possible contributing factor is that the frequency of laboratory testing for respiratory pathogens decreases with age.

Our results suggest that RSV infection is common in older children and is an important driver of the often serious ALRI episodes observed in infants. The fact that RSV remains largely undetected in older children indicates that infection may be asymptomatic or does not result in symptoms severe enough to warrant medical attention. This is consistent with findings from household studies $[16,17]$ where parents and siblings of a young child with RSV infection who were RSV-positive themselves often displayed no symptoms.

There are several limitations to this study. Our data does not provide us with information about the ages of older siblings and therefore we were not able to estimate how much of the excess risk is attributable to children attending school who may be the most effective targets for vaccination to protect the newborn. A population-based study such as this using administrative data from Western Australia can only capture the more severe cases and only 9\% of RSV detections arose from non-hospital settings. At present there is no population-based primary-care database which could provide valuable information about risk factors for a wider severity spectrum of RSV infections. Another limitation is that, due to missing data, we have not presented results from models which adjust for the potential confounding effect of socioeconomic status. However, we did undertake an adjusted 


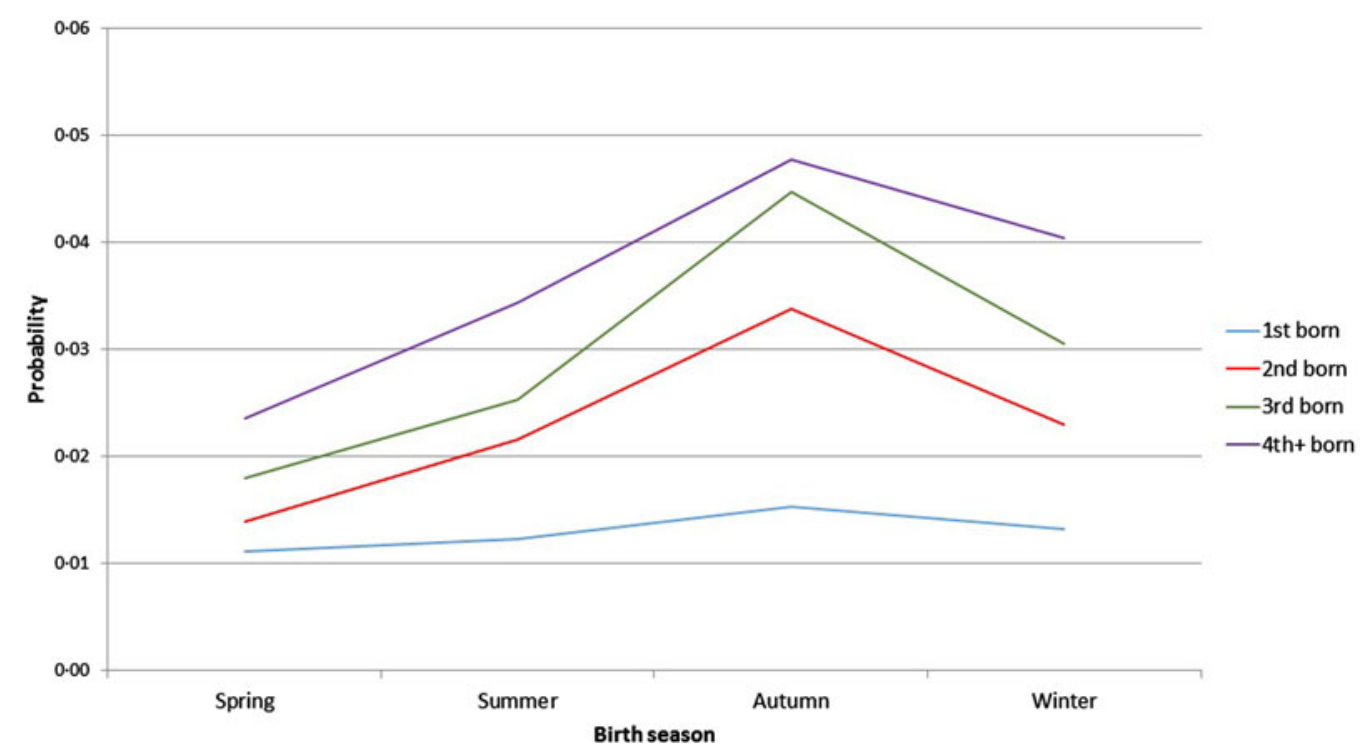

Fig. 1. Fitted probabilities* of respiratory syncytial virus detection in infants by birth order and birth season [* estimated marginal means from models adjusted for gender, Aboriginal status, preterm birth $(<37$ weeks), birthweight, maternal age (five groups) and gestational smoking].

analysis using those records where area-level socioeconomic status was available $(\sim 80 \%$ of infants in our cohort) and this showed very little change to the sibling effects we have reported.

Although vaccine development is proceeding rapidly and has been a priority for some years, a licensed product has yet to emerge [25]. Our results support the view [26] that vaccine development efforts should also be directed towards an alternative or supplementary strategy to vaccinating the RSV-naive infant which would involve routine vaccination of older siblings to reduce the indirect risk of a newborn developing an acute RSV-related illness during infancy. We will use these results to further our development of mathematical models investigating the seasonality and transmission of RSV with a view to assessing the impact of potential vaccination strategies.

\section{ACKNOWLEDGEMENTS}

H.C.M. is supported by National Health and Medical Research Council Fellowship APP1034254. The use of linked data was approved by the Western Australia Data Linkage Branch at the Western Australian Department of Health and the authors are grateful for their ongoing support.

\section{DECLARATION OF INTEREST}

None.

\section{REFERENCES}

1. Nair H, et al. Global burden of acute lower respiratory infections due to respiratory syncytial virus in young children: a systematic review and meta-analysis. Lancet 2010; 375: 1545-1555.

2. Lanari M, et al. Risk factors for bronchiolitis hospitalization during the first year of life in a multicenter Italian birth cohort. Italian Journal of Pediatrics 2015; 41: 40

3. Papadopoulos NG, et al. Association of rhinovirus infection with increased disease severity in acute bronchiolitis. American Journal of Respiratory and Critical Care Medicine 2002; 165: 1285-1289.

4. Moore HC, et al. Modelling the seasonal epidemics of respiratory syncytial virus in young children. $P L O S$ ONE 2014; 9: e100422.

5. Simoes EA. RSV disease in the pediatric population: epidemiology, seasonal variability, and long-term outcomes. Managed Care 2008; 17: 3-6, discussion 18-19.

6. Falsey AR, et al. Respiratory syncytial virus and other respiratory viral infections in older adults with moderate to severe influenza-like illness. Journal of Infectious Diseases 2014; 209: 1873-1881.

7. Hall CB, Long CE, Schnabel KC. Respiratory syncytial virus infections in previously healthy working adults. Clinical Infectious Diseases 2001; 33: 792-796.

8. Hall CB, et al. Respiratory syncytial virus-associated hospitalizations among children less than 24 months of age. Pediatrics 2013; 132: e341-348.

9. Bourgeois FT, et al. Influenza and other respiratory virus-related emergency department visits among young children. Pediatrics 2006; 118: e1-8.

10. Mullard A. Making way for maternal immunization. Nature Reviews Drug Discovery 2016; 15: 3-4. 
11. Leader S, Kohlhase K. Recent trends in severe respiratory syncytial virus (RSV) among US infants, 1997 to 2000. Journal of Pediatrics 2003; 143: S127-132.

12. Law BJ, Carbonell-Estrany X, Simoes EA. An update on respiratory syncytial virus epidemiology: a developed country perspective. Respiratory Medicine 2002; 96 (Suppl. B): S1-7.

13. Hall CB, et al. The burden of respiratory syncytial virus infection in young children. New England Journal of Medicine 2009; 360: 588-598.

14. Simoes EA. Environmental and demographic risk factors for respiratory syncytial virus lower respiratory tract disease. Journal of Pediatrics 2003; 143: S118-126.

15. Munywoki PK, et al. The source of respiratory syncytial virus infection in infants: a household cohort study in rural Kenya. Journal of Infectious Diseases 2014; 209: 1685-1692.

16. Heikkinen T, et al. Transmission of respiratory syncytial virus infection within families. Open Forum Infectious Diseases 2015; 2: ofu118.

17. Crowcroft NS, et al. Respiratory syncytial virus infection in infants admitted to paediatric intensive care units in London, and in their families. European Journal of Pediatrics 2008; 167: 395-399.

18. Murray $\mathbf{J}$, et al. Risk factors for hospital admission with RSV bronchiolitis in England: a population-based birth cohort study. PLoS ONE 2014; 9: e89186.

19. Kelman CW, Bass AJ, Holman CD. Research use of linked health data - a best practice protocol. Australian and New Zealand Journal of Public Health 2002; 26: 251-255.

20. Bradley JS, et al. The management of community-acquired pneumonia in infants and children older than 3 months of age: clinical practice guidelines by the Pediatric Infectious Diseases Society and the Infectious Diseases Society of America. Clinical Infectious Diseases 2011; 53: e25-76.

21. Moore HC, et al. A retrospective population-based cohort study identifying target areas for prevention of acute lower respiratory infections in children. $B M C$ Public Health 2010; 10: 757.

22. Moore HC, et al. Use of data linkage to investigate the aetiology of acute lower respiratory infection hospitalisations in children. Journal of Paediatrics and Child Health 2012; 48: 520-528.

23. Rockhill B, Newman B, Weinberg C. Use and misuse of population attributable fractions. American Journal of Public Health 1998; 88: 15-19.

24. Lim FJ, et al. Optimization is required when using linked hospital and laboratory data to investigate respiratory infections. Journal of Clinical Epidemiology 2016; 69: 23-31.

25. Anderson LJ, et al. Strategic priorities for respiratory syncytial virus (RSV) vaccine development. Vaccine 2013; 31 (Suppl. 2): B209-215.

26. Graham BS. Protecting the family to protect the child: vaccination strategy guided by RSV transmission dynamics. Journal of Infectious Diseases 2014; 209: 1679-1681. 